\title{
A Dempster-Shafer approach for recognizing machine features from CAD models
}

\author{
Qiang Ji ${ }^{\mathrm{a}, *}$, Michael M. Marefat ${ }^{\mathrm{b}}$ \\ ${ }^{a}$ Department of Electrical, Computer, and Systems Engineering, Rensselaer Polytechnic Institute, JEC 6003, Troy, \\ NY 12180-3590, USA \\ ${ }^{\mathrm{b}}$ Department of Electrical and Computer Engineering, University of Arizona, Tucson, AZ 85721, USA
}

Received 19 November 2001; received in revised form 28 May 2002; accepted 6 August 2002

\begin{abstract}
This paper introduces an evidential reasoning-based approach for recognizing and extracting manufacturing features from solid model description of objects. A major difficulty faced by previously proposed methods for feature extraction has been the interaction between features due to non-uniqueness and ambiguousness in feature representation. To overcome this difficulty, we introduce a Dempster-Shafer approach for generating and combining geometric and topologic evidences to identify and extract interacting features.

The main contributions of this research include introducing different classes of evidences based on the geometric and topologic relationships at different abstraction levels for effective evidential reasoning and developing the principle of association to overcome the mutual exclusiveness assumption of the Dempster-Shafer theory. Experiments demonstrate the effectiveness of the proposed approach in extracting interacting machine features.
\end{abstract}

(C) 2003 Pattern Recognition Society. Published by Elsevier Science Ltd. All rights reserved.

Keywords: Dempster-Shafer theory; Evidential reasoning; Feature extraction; Pattern recognition; Geometric reasoning

\section{Introduction}

Traditional CAD boundary description of a solid object in terms of low level entities like faces, edges, and vertices is not conducive to manufacturing automation. Most machine operations require high level semantic features such as pockets, slots, and holes instead of these low level geometric entities. Therefore, in order to achieve true manufacturing automation, several different mechanisms have been proposed to automatically extract the high level semantic features from the low level entities in solid model representations [1-11]. While these mechanisms can successfully recognize decoupled or isolated primitive features, they have achieved very limited success in identifying and describing

\footnotetext{
* Corresponding author. Tel.: +1-775-784-6974; fax: +1-775-784-1877.

E-mail address: qji@ecse.rpi.edu (Q. Ji).
}

compound depressions generated by the interaction of several semantic features [5,12-14].

The difficulty in extracting interacting features arises from the ambiguous and non-unique feature representation schemes. A representation scheme is unambiguous if each of its valid representations corresponds to a single entity in its domain. A representation scheme is unique if all entities in its domain admit only one syntactically correct representation. Unfortunately, most representation schemes currently available are not unique or unambiguous in a general sense. The ambiguity of feature representations is one of the major sources of difficulty for feature extraction mechanisms. The reason is that regardless of the actual workings of the mechanism (say expert rules, syntactic patterns, or graph isomorphism), the employed mechanism searches for a particular combination of geometric and topologic relationships to recognize individual primitive features. However, as outlined above, due to the representation ambiguities, 


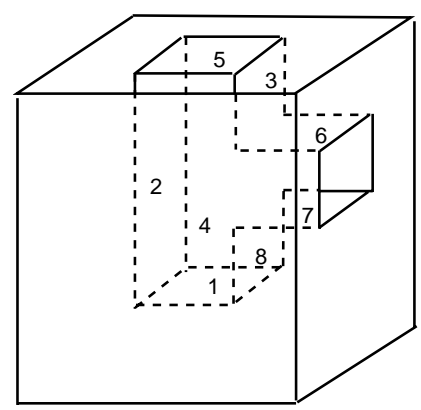

(a1)

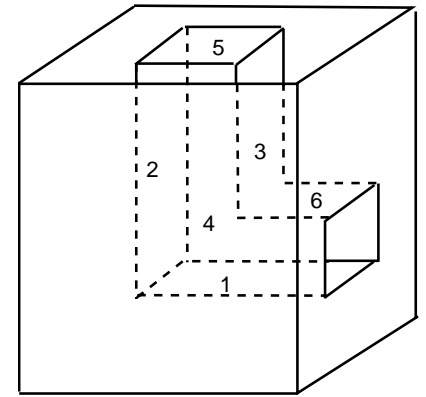

(b1)
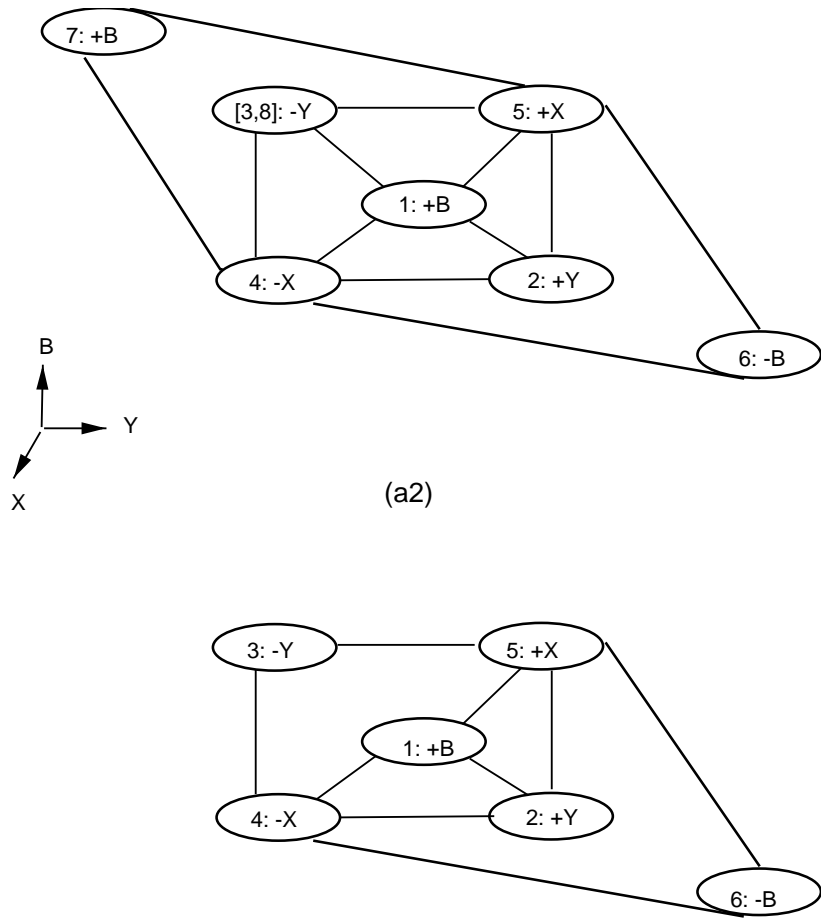

(b2)

Fig. 1. Two parts (a1 and b1) with two perpendicular pockets of identical size and orientation, and their cavity graph representations (a2 and $\mathrm{b} 2$ ).

these geometric and topologic relationships are not unique, and therefore the feature recognition mechanism produces brittle behavior.

Let us illustrate the point by means of an example. Graph-based mechanisms, typically, represent a part (or its depression) by a graph (for example a node for each face, and a link between two nodes whose corresponding faces share an edge), and each primitive feature by its corresponding template graph. Subsequently, they use a method like graph isomorphism to identify instances of the features in a given part. For example, Fig. 1 (a1 and b1) shows two parts with two perpendicular pockets of identical size and orientation. The cavity graph representations (see Fig. 2 for the definition of cavity graph) of the depressions for both parts are shown in Fig. 1(a2) and (b2). Furthermore, Fig. 1 shows that although the rectangular pocket formed by the nodes $1-5$ in Fig. 1(a2) may be extracted by the pocket template (see the first row in Fig. 2), the rectangular pocket formed by the same set of nodes in Fig. 1(b2) cannot be correctly identified as the pocket primitive due to lack of a link between nodes 3 and 1 . This is because the topologic relationships of the two parts are different.

Let us also consider the same problem from an expert rule-based view. A typical rule to recognize the class of pockets could look like the following:

(Pocket List-of-faces: $\{\mathrm{A}\}$ ) IF

(A base-face: $b$ is a member of $\{A\}$ ) AND

(base-face: $b$ is concavely adjacent to all sides $\{A\}-\{b\}$ ) AND

(each side face in $\{A\}-\{b\}$ is concavely adjacent to 2 other sides in $\{A\}-\{b\})$.

In simple words, the above rule says that a list of faces forms a pocket, if there is a base face in the group of faces which is concave to every other face, and if every non-base (or side) face is concavely adjacent to two other neighboring side faces. It is again not difficult to show that such a rule-based approach works all right when the features are decoupled (e.g. Fig. 1(a)), but it will produce brittle behavior for other cases. For example, in the part depicted in Fig. 1(b), the base-face, i.e. face 1 , is not concavely adjacent to one of the sides, i.e. side-face 3 , and therefore, the second antecedent in the above rule will evaluate to false. The above rule fails to recognize the pocket formed by faces $1-5$ in Fig. 1(b1). The reason is that rules, such as the one shown, attempt to capture generic topologic and geometric relationships indicative of different classes of features, but instances of the features may not follow the captured 


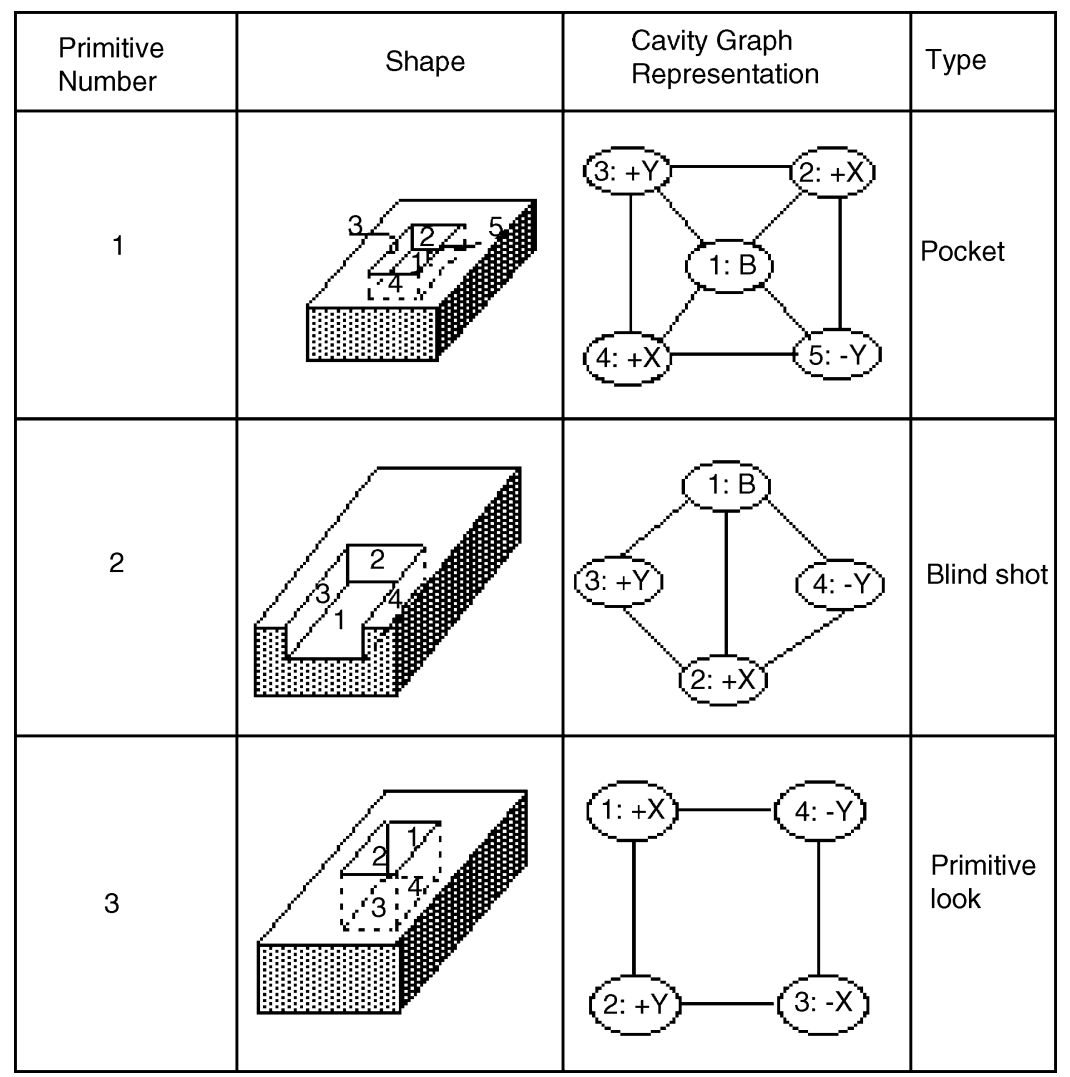

Fig. 2. Some families of primitive features and their cavity graph representations. For cavity graph, faces are represented by nodes and concave adjacency relationships between faces are represented by links. Node labels are the dominant spatial directions $(X, Y$, and $B$ as defined in a local coordinate system as shown in Fig. 1) of surface normals of the corresponding faces. Other consistent permutations of $X, Y$, and $B$ also lead to the representations for the same primitive.

relationships (especially when a feature interacts with other features).

The set of topologic and geometric relationships which describes a class of features is not unique. One method to overcome this non-uniqueness is to attempt to enumerate all possible topologic and geometric relationships which describe instances of a given class of features under all different circumstances. Such an attempt, however, would either be impossible or exceedingly difficult because of the difficulty in enumerating all distinct configurations, which are either infinite or very large. Instead of the above brute force enumeration method, the approach that we propose in this paper is to combine evidences in a well defined manner to determine features present in a depression.

Evidences supporting or rejecting existence of instances of different features can be combined to determine how depressions of a part can be described in terms of primitive manufacturing features such as pockets and slots. In such a framework, each evidence may not by itself be sufficient for recognizing a feature, but it generates a belief (or a weight), which is a measure of confidence that relates the evidence (a topologic or geometric relationship) to a feature. It is the collection of evidences and their consistent impacts upon each other that produces a description of the features in a depression.

There are several important advantages to such a scheme for extracting and identifying features including: (i) since the features in the depression are recognized by the collection of the evidences, the individual evidences may or may not carry equal weight, and in fact some of the individual evidences may support conflicting conclusions; as long as their cumulative combination is in favor of a correct interpretation, a correct description of the depression will be obtained, (ii) one does not need to know ahead of time which set of topologic and geometric relationships, or which patterns, will be observed for each feature, in fact at different times different topologic and geometric relationships may indicate the same feature class, and (iii) the same topologic or geometric relationship may simultaneously support two different features with different degrees of confidence.

The primitive features of interest in this work are common polyhedral machinable features like pockets, blind slots, 
prismatic holes, and steps. The primitive features used in this paper have faces which are concave to each other, however extension to features with both convex and concave relationships can be addressed similarly. Fig. 2 shows instances of some families of primitive features considered in this research along with their cavity graph representations.

\section{Literature review}

Various approaches have been proposed for automatic feature extraction from CAD solid models. These methods can be classified into the following categories: syntactic pattern recognition [15-18], graph-based methods [19-23], rule-based methods [1,24], and probabilistic or evidential reasoning methods $[5,25,26]$. Syntactic pattern recognition characterizes the overall part shapes as the composition of certain geometric primitives. Feature recognition proceeds by parsing the input syntactic expression of a part using grammar rules to identify the syntactic patterns representing the geometric primitives. In graph-based feature recognition, a graph is used to describe the topological shape of a part as well as that of a primitive feature. Feature recognition is performed by searching graph representation of the part to identify a subgraph that matches that of a primitive using subgraph isomorphism. The idea of the rule-based method for feature extraction is that rules are used to capture the knowledge about geometric and topological properties of form features. Features are recognized on the basis of certain pre-specified rules that are characteristic to the features.

In addition to the problem inherited from pattern-matching, each of the three approaches has the following problems. For graph-based method, extensive pre-processing is required to construct graphic representation for each part and each primitive. Its application is limited to the polyhedral parts. For syntactic method, its application is limited to 2D prismatic parts, rotational parts with turning features and any axis symmetric volumes, but success for non-axis symmetric 3D part or rotational parts with non-turning features has been very limited. Also pure syntactic representation seems too restrictive. Finally, for rule-based method, it is a generalization of syntactic method and has proved to be more robust and handling more kinds of parts than syntactic method. Its shortcomings include ambiguous representation, rules for every conceivable feature, and verbose.

In view of these problems, a new approach based on evidential reasoning was introduced to recognize features from CAD models. Evidential reasoning performs feature recognition by gathering the available geometric and topological evidences and performing uncertainty reasoning based on the evidences collected to recognize features. Evidential reasoning or uncertainty reasoning offers a consistent means for handling conflicting, redundant, or missing data. While features interacting, uncertainty develops as a result of non-uniqueness of the patterns associated with the topology and geometry of features in these interactions. Despite of uncertainty, there is also available abundance of geometric and topologic evidences, which if used correctly, can lead to the resolution of the uncertainty and therefore eventual recognition of the features. Uncertainty reasoning offers a formal and consistent mechanism to accomplish this goal through evidence aggregation.

The two most popular techniques for handling uncertainties are the Bayesian theorem [27] and the Dempster-Shafer theorem [28]. While the former is a probabilistic reasoning process, the latter is an evidential reasoning process. In the former, the relationships between evidences and hypotheses are described by three numbers: prior probability, likelihood, and posterior probability. Evidences are combined using the Bayesian theorems. In the later, the relationships between evidences and hypotheses are described in terms of belief intervals and basic probability assignment. Evidences are combined using its rule of combination.

In the filed of feature extraction from CAD models, Marefat [5] first introduced uncertainty reasoning in analyzing interacting features. He proposed the use of Dempster-Shafer method to recover the missing links lost due to feature interaction. The missing links are identified from a set of possible missing links by accumulating both geometric and topological evidences using Dempster's rule of combination. Due to mutual-exclusiveness assumption with the DS theory, their method, however, is limited to recover one missing link at a time, therefore is computationally inefficient. Ji [26] further expanded Marefat's original approach using Bayes networks. The expanded method can simultaneously recover all missing links and recognize more complex interacting features. In addition, a new contribution of this method is the generation and incorporation of hierarchical feature-based evidence, that can significantly improve the recognition accuracy. Trika and Kashyap [25] proposed a feature-recognition similar to the work of Ji.

To be complete, we would also like to point out that the research presented in this paper resembles the earlier and well known work by David Waltz [29] on recognizing features based on edges or junctions/vertices via constraint satisfaction since we are also dealing with the block features. To recognize objects, Waltz [29] developed a method based on a junction labeling scheme that associates certain labels/attributes (e.g., positive, negative, concave, convex, Fork junction, T junction) with each edge or junction. The geometric constraints imposed on the adjacent edges/junctions are propagated to all edges/vertices of the objects. A valid object is recognized if all its edges/vertices satisfy the geometric constraints and are consistent with each other. A similar algorithm was later proposed by Mackworth [30]. It appears that this classic work is applicable to our work since we are also recognizing features consisting of edges, faces, and junctions. A careful study reveals that Waltz's work is difficult to apply however.

First, though we try to recognize block features, we are not directly dealing with block objects. In fact, we try to recognize features from their solid model representations 
(e.g. boundary representation). The junctions/vertices, though included in the representation, do not have the necessary labels information such as positive/negative, Arrow junction, $\mathrm{T}$ junction, etc. as proposed by Waltz to classify junctions into different categories. Lacking such information makes it difficult to automatically use Waltz's method to limit the search space for virtual links unless such information can be derived manually. Second, for our method, feature recognition is performed on the cavity graph that we use to represent features. Cavity graph consists of only edges and surfaces. To incorporate junctions/vertices into the cavity graph requires significant modification of the cavity graph representation, which is not a trivial task. Finally, Waltz's work does not deal with feature recognition with incomplete information. Our algorithm specifically focuses on recognizing the features under incomplete information, i.e., in the absence of some surfaces (links) due to feature interaction. Our method determines, based on pooling various geometric evidences, if those missing surfaces should be used to infer the presence of a geometric feature.

In summary, evidential reasoning is an especially wellsuited approach to feature recognition. It systematically combines the available geometric and topological evidences to provide a feature interpretation that best explains the available evidences. In the sections to follow, we will expand our previous work in feature recognition based on the Dempster-Shafer theory to allow more efficient and accurate feature recognitions from CAD models.

\section{Problem formulation and solution overview}

\subsection{Brief overview of graph-based approach}

Let us more closely consider the graph-based approach to feature recognition and extraction. The following definitions are needed for this purpose:

Definition 1. A global graph $G$ for an object $S$ is an ordered four tuple $\left(N(G), L(G), \Psi_{G}, l_{G}\right)$ with the following properties.

1. $N(G)$ is a non-empty set of nodes of $G$, such that for each face $f_{i}$ of the object there is exactly one node in $G$.

2. $L(G)$ is the set of links of $G$, such that for every edge $e_{i j}$ of the object shared by two faces $f_{i}$ and $f_{j}$, there is a link connecting the corresponding nodes in $G$.

3. $\Psi_{G}: L(G) \rightarrow N(G) \times N(G)$ is an incidence function that associates with every link of $G$ an unordered pair of nodes of $G$.

4. $l_{G}: L(G) \rightarrow\{$ concave, convex $\}$ is a function which labels the links of $G$. This labeling marks a link in the graph concave if the faces sharing the corresponding edge of the object are concavely adjacent, and marks the link convex otherwise.
Definition 2. A cavity graph $G^{\prime}$ for a depression is an ordered five tuple $\left(N^{\prime}\left(G^{\prime}\right), L^{\prime}\left(G^{\prime}\right), \Psi_{G^{\prime}}^{\prime}, l_{G^{\prime}}^{\prime}, m_{G^{\prime}}^{\prime}\right)$ such that it is connected and:

1. $N^{\prime}\left(G^{\prime}\right) \subseteq N(G)$ is the set of nodes of $G^{\prime}$ such that the unifiable nodes of $G$ are unified [5],

2. $L^{\prime}\left(G^{\prime}\right)$ is the set of links of $G^{\prime}$ such that $l_{G}(k)=$ concave $\forall k \subseteq l^{\prime}\left(G^{\prime}\right)$, that is every link has a concave label,

3. $\Psi_{G^{\prime}}^{\prime}: L^{\prime}\left(G^{\prime}\right) \rightarrow N^{\prime}\left(G^{\prime}\right) \times N^{\prime}\left(G^{\prime}\right)$, and $l_{G^{\prime}}^{\prime}: L^{\prime}\left(G^{\prime}\right) \rightarrow$ \{concave, convex $\}$ are the incidence which define the incidence function and the link labels as before, and

4. $m_{G^{\prime}}^{\prime}: N^{\prime}\left(G^{\prime}\right) \rightarrow\{B,-B,+X,-X,+Y,-Y\}$ is a function which labels the nodes of $G^{\prime}$. This labeling of the nodes describes the relative spatial orientation of the faces with respect to each other in a qualitative manner [5].

Since depressions are usually associated with concavities in a component, the cavity graphs, which consists solely of concave links, are intended to model the depressions of an object. Fig. 1(b2) shows the cavity graph for the depicted part which has two perpendicularly interacting pockets (a part may have more than one cavity graph). As briefly stated before, in a typical graph-based approach [2,5], a part or its depressions are represented by graphs, and subsequently a matching between this representation and the feature templates is used for recognition. For example, in the cavity graph shown in Fig. 1(a2), the center nodes and links correspond to a pocket template (see the first row in Fig. 2), which is formed by faces 1-5. In general, this method can be stated as follows: given a cavity graph $G^{\prime}$, find a decomposition, $D=\left\{g_{1}, g_{2}, \ldots, g_{n}\right\}$, such that:

(i) $g_{i}$ are primitive templates,

(ii) $g_{1} \cup g_{2} \cup \cdots \cup g_{n}=G^{\prime}$

(iii) $g_{i}$ are maximal, that is $g_{i} \subseteq g_{j}$ for $i>j$.

Therefore, in this method, a partitioning of the cavity graph(s) is used to obtain a part's features. The partitions may overlap, but the maximality condition in (iii) above ensures that for example a pocket is not mistaken for two blind-slots, etc. In our earlier work [5], we described detailed methods to generate the correct partitioning, $\left\{g_{1}, g_{2}, \ldots, g_{n}\right\}$, from a cavity graph, $G^{\prime}$.

\subsection{Virtual links}

In the previous section, we discussed the use of partitioning to determine the features of a part. However, a correct partitioning of the cavity graphs may still not result in the valid templates for the features of the part, because of the non-uniqueness in representations. For example, Fig. 3 shows the partitioning for the cavity graph shown in Fig. 1(b2).

It is clear that neither one of the subgraphs produced from the partitioning corresponds to a pocket (see pocket representation in Fig. 2). In order to identify and extract the 


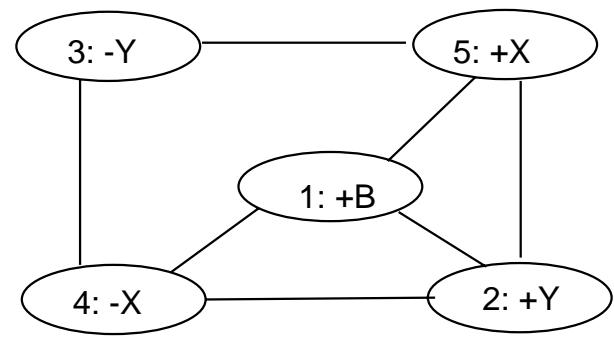

(a)

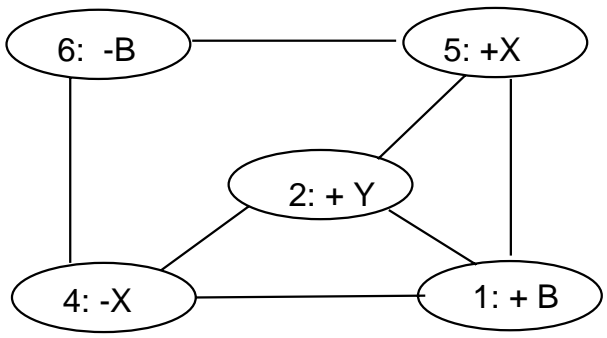

(b)

Fig. 3. Cavity graph partitioning (a) and (b) are the subgraphs of the cavity graph shown in Fig. 1(b2) corresponding to the two pockets of the involved part. However, neither (a) nor (b) matches the template for a pocket primitive, because in (a), the link 3-1 is needed before the representation matches a pocket template, and in (b), the link 6-2 is needed to obtain a pocket template.

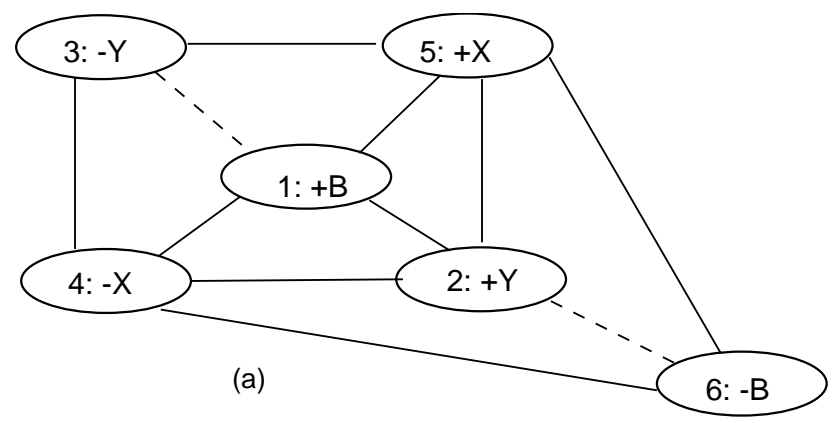

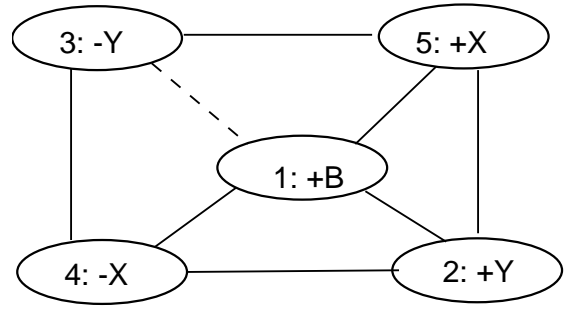

(b1)

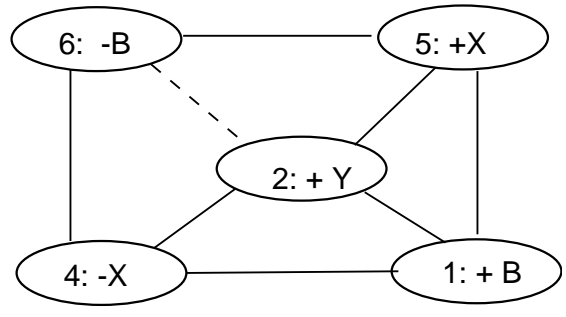

(b2)

(b)

Fig. 4. Cavity graphs with virtual links: (a) shows the cavity graph for the example part shown in Fig. 1(b2) augmented with the virtual links 3-1 and 6-2. The augmented cavity graph is readily partitioned into two maximal constituents, (b1) and (b2), each of which represents one of the pockets in the depression of the part.

two pockets for the part in Fig. 1(b1), the cavity graph in Fig. 1(b2) must be augmented with two links, 3-1 and 6-2, and then correctly partitioned. The new cavity graph produced by augmenting the original cavity graph with these two links is shown in Fig. 4(a), and the subgraphs which result from its partitioning are shown in Fig. 4(b). It is clear that the subgraphs in Fig. 4(b) correctly identify the pockets in the depression of the given part.

Definition 3. Virtual links: Suppose a depression with cavity graph $G^{\prime}$ consists of the features $f_{1}, f_{2}, \ldots, f_{n}$, with the corresponding representations $g_{1}, g_{2}, \ldots, g_{n}$; then the set of links of $\left\{g_{1} \cup g_{2} \cup \cdots \cup g_{n}\right\}-G^{\prime}$ are referred to as virtual links (provided that this set is not empty).

In other words, virtual links are set of links which are not present in the cavity graph of a depression, but whose augmentation results in a supergraph which is isomorphic to the union of the representations for the involved features, and hence can be partitioned to obtain these features. In general, the number of required virtual links as well as their identity for a given depression are not known in advance. Therefore the problem of combining different geometric and 


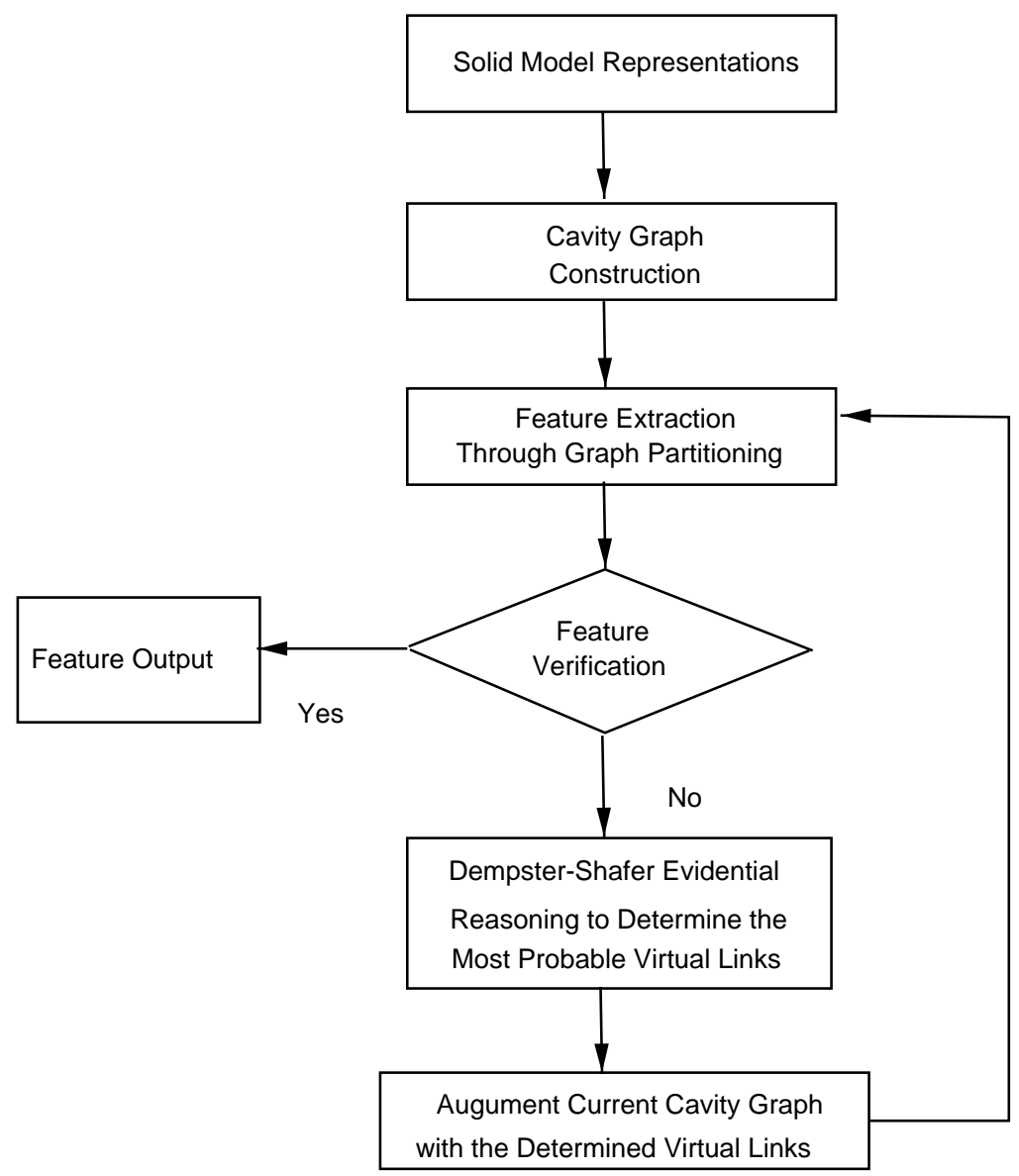

Fig. 5. Schematic diagram of the proposed approach based on evidential reasoning and graph partitioning to identify and extract features.

topologic evidences to determine the features can be stated as follows in terms of virtual links and partitioning.

Problem statement: Given a depression, generate and combine different appropriate evidences based on the existing geometric and topologic relationships to determine the necessary virtual links, augment the existing cavity graph with the determined virtual links, and then partition the resulting cavity graph to extract the features.

Fig. 5 shows the schematic diagram for the overall solution approach. The algorithm starts with the construction of a cavity graph for the depression of a given part from its solid model representation. Following the cavity graph construction, the algorithm proceeds to perform a graph partitioning over the cavity graph to determine whether the current cavity graph can be decomposed into subgraphs representing embedded primitive features. Due to feature interaction, the resulting subgraphs may not correspond to the desired primitive features. The Dempster-Shafer reasoning is subsequently activated to recover the virtual links. Geometric and topologic evidences are aggregated to determine the most probable set of links as virtual links. The original cavity is then augmented with the determined virtual link(s) and graph partitioning the reconstructed cavity graph results in a set of subgraphs that represent the embedded interacting features.

\section{Feature extraction using Dempster-Shafer theory}

\subsection{The Dempster-Shafer theory}

The Dempster-Shafer (D-S) approach [31] offers a systematic and consistent mechanism to represent and propagate uncertainties in a coherent manner by aggregating the available geometric and topologic evidences. According to the D-S theory, the set of all possible outcomes (the sample space) in a random experiment is called the frame of discernment (FOD) denoted by $\theta$. For example, if we toss a fair coin twice, the set of all outcomes could be described as $\{h h, h t, t h, t t\}$, where ' $h$ ' and ' $t$ ' represent the head and tail of the coin respectively. The frame of discernment is therefore $\theta=\{h h, h t, t h, t t\}$. Subsets of $\theta$ are referred to as events 
or hypotheses. A major assumption in D-S theory is that elements of $\theta$ must be mutually exclusive and exhaustive to each other. This assumption implies that one and only one element in $\theta$ could be correct. Later, we will demonstrate the limitation of this assumption and propose a method to overcome this limitation.

According to the $\mathrm{D}-\mathrm{S}$ theory, a number in the range $[0,1]$ inclusive is used to indicate the belief in a hypothesis (or a set of hypotheses) given a piece of evidence. This number, referred to as basic probability assignment (bpa), expresses the degree to which an evidence confirms a hypothesis. It measures the belief assigned to the hypothesis from the evidence.

Given two bpa's $m_{1}()$ and $m_{2}()$ discerned in the same frame, their combined effect can be computed using the Dempster's rule of combination as

$m(C)=m_{1} \oplus m_{2}(C)=K \sum_{A \cap B=C} m_{1}(A) m_{2}(B)$,

where

$K^{-1}=1-\sum_{A \cap B=0} m_{1}(A) m_{2}(B)$.

The combination rule states that representing the combination of $m_{1}$ and $m_{2}$ apportions the total amount of belief among the subsets of $\theta$ by assigning $m_{1}(A) m_{2}(B)$ to the set resulting from intersection of sets $A$ and $B . K$ is a normalization constant.

\subsection{Identifying virtual links one at a time}

A major assumption in D-S theory is that elements of $\theta$ must be mutually exclusive and exhaustive. This assumption implies that one and only one element in $\theta$ could be the correct outcome. Hence, given a frame of discernment containing all potential virtual link, the assumption means that one and only one potential virtual links can be a virtual link. This assumption is too restrictive since it is possible to have multiple virtual links. To overcome this limitation, we propose an approach that employs an iterative feedback loop during the evidence aggregation process, with one virtual link being determined at a time. This approach continues iteratively until graph partitioning of the latest cavity graph results in subgraphs that represent the union of the involved primitives. While observing the mutual exclusiveness assumption, this approach manages to identify all virtual links. The example part shown in Fig. 1(b) will be used to explain the proposed approach.

\subsubsection{Construction of the frame of discernment}

To identify virtual links, a hypothesis space is required. In this research, the hypothesis space $H$ should contain all the potential virtual links. Hence, elements of $H$ consist of links in the complement of the cavity graph. For example, for the cavity graph shown in Fig. 1(b2), the six links in the complement of the cavity graph form a hypothesis space $H=$ $\left\{f_{1} f_{3}, f_{1} f_{6}, f_{2} f_{3}, f_{2} f_{6}, f_{3} f_{6}, f_{4} f_{5}\right\}$, where $f_{m} f_{n}$ represents a link between node $m$ and node $n$ in the cavity graph.

Given the hypothesis space, a frame of discernment can be constructed. The frame of discernment $\theta$ should contain all possible potential virtual links. For the example part shown in Fig. 1(b2), we have:

$\theta=H=\left\{f_{1} f_{3}, f_{1} f_{6}, f_{2} f_{3}, f_{2} f_{6}, f_{3} f_{6}, f_{4} f_{5}\right\}$.

\subsubsection{Evidence generation}

To identify virtual links, evidences are required. Evidences, which describe topologic and geometric relationships, support or disconfirm hypotheses in the hypothesis space through a measure of confidence or a basic probability assignment (bpa). Each evidence by itself may not be sufficient to irrefutably confirm or disconfirm subsets of the frame of discernment, but the collective knowledge of these evidences should provide us strong indication about the correct choices. In this approach, we have developed face-based evidences for employment in the above frame of discernment (Eq. (2)).

Face-based evidences consider particular geometry and topology between a pair of faces and relate the particular observed geometric and topologic relationship to the possible existence of a virtual link between the given pair of faces. For example, it is more probable to have a concave intersection and hence a virtual link between a pair of planar faces which are nearly perpendicular, than between a pair of planar faces which are nearly parallel. Therefore, observation of surface perpendicularity in the CAD solid model description of the faces can be used to assign a higher belief to the hypothesis for a virtual link between them. Similarly, convexity between two faces is an evidence that strongly disconfirms the existence of a virtual link between the involved faces. For example, a higher bpa value is assigned to a link with two concave faces than to a link with convex faces. Specifically, the bpa assignments are based on the following rules.

- If two faces $f_{i}$ and $f_{j}$ have orthogonal principle normals, assign $m\left(\left\{\left(f_{i} f_{j}\right)\right\}\right)=0.7$.

- If two faces $f_{i}$ and $f_{j}$ are concavely adjacent to each other, assign $m\left(\left\{\left(f_{i} f_{j}\right)\right\}\right)=0.7$.

- If two faces $f_{i}$ and $f_{j}$ are convexly adjacent to each other, assign $m\left(\left\{\left(f_{i} f_{j}\right)\right\}^{c}\right)=0.8$. (c here stands for complement).

- If two faces $f_{i}$ and $f_{j}$ have almost parallel principle normals, assign $m\left(\left\{\left(f_{i} f_{j}\right)\right\}^{c}\right)=0.8$.

Based on the above rules, the BPA assignments for the example part shown in Fig. 1(b) can be summarized in Table 1.

\subsubsection{Experimental results}

Given the frame in Eq. (2), the Dempster's rule of combination was then used to aggregate the face evidences in 
Table 1

BPA assignments by face-based evidences for the example part

\begin{tabular}{ll}
\hline$m_{1}\left(f_{1} f_{3}\right)=0.7$ & $m_{1}(\theta)=0.3$ \\
$m_{2}\left(f_{1} f_{6}\right)^{c}=0.7$ & $m_{2}(\theta)=0.3$ \\
$m_{3}\left(f_{2} f_{3}\right)^{c}=0.7$ & $m_{3}(\theta)=0.3$ \\
$m_{4}\left(f_{2} f_{6}\right)=0.7$ & $m_{4}(\theta)=0.3$ \\
$m_{5}\left(f_{3} f_{6}\right)=0.7$ & $m_{5}(\theta)=0.3$ \\
$m_{6}\left(f_{3} f_{6}\right)^{c}=0.8$ & $m_{6}(\theta)=0.2$ \\
$m_{7}\left(f_{4} f_{5}\right)^{c}=0.7$ & $m_{7}(\theta)=0.3$ \\
\hline
\end{tabular}

Table 2

Combined BPAs for the example part shown in Fig. 1(b)

\begin{tabular}{ll}
\hline Links & Combined BPAs \\
\hline$\left\{f_{1} f_{3}\right\}$ & $\mathbf{0 . 3 6 6}$ \\
$\left\{f_{6} f_{2}\right\}$ & $\mathbf{0 . 3 6 6}$ \\
$\left\{f_{3} f_{6}\right\}$ & 0.110 \\
$\left\{f_{4} f_{5}\right\}$ & 0.000 \\
$\left\{f_{3} f_{2}\right\}$ & 0.000 \\
$\left\{f_{6} f_{1}\right\}$ & 0.000 \\
\hline
\end{tabular}

Table 1. Table 2 gives the aggregated beliefs in each of the six potential virtual links.

Links with highest beliefs are shown in bold in Table 2. They are links $\left\{f_{3} f_{1}\right\}$ and $\left\{f_{6} f_{2}\right\}$. Due to the mutual exclusiveness assumption, only one of the six link can be a virtual link. If we choose any one of the two and add it back to the original cavity graph, partitioning the newly modified cavity graph still cannot result in subgraphs representing correct primitive features, which means the need for additional virtual links. Repeating the above process until the latest partitioned cavity graph can result in subgraphs representing the embedded primitive features. For our example, this process repeats twice with two virtual links $\left\{f_{3} f_{1}\right\}$ and $\left\{f_{6} f_{2}\right\}$ identified consecutively. The latest augmented cavity graph is shown in Fig. 4(a), where the two augmented virtual links are shown by dotted lines. Partitioning the modified graph identifies two pockets, one with base-face 1 and side-faces $2-5$, and the other with base-face 2 and side-faces $1,4,5,6$, as form features of this object. This is the correct interpretation for the example part.

\subsection{Identifying multiple virtual links}

One obvious disadvantage with the above approach is that it cannot determine multiple virtual links simultaneously due to the mutual exclusiveness assumption. It needs several iterations to recover all necessary virtual links. For each iteration, it constructs a hypothesis space, frame of discernment, and then performs evidences-gathering and aggregation. This greatly increases the computational complexity. In this section, we propose a method for identifying mul- tiple virtual links simultaneously while still observing the mutual exclusiveness assumption.

\subsubsection{Construction of the frame of the discernment}

Dealing with multiple cause sources, i.e., the simultaneous presence of several correct hypotheses, adds considerable complexity to the inference process and problem formulation due to the mutual exclusiveness constraint. However, several approaches have been suggested to address this issue. One way to overcome this difficulty is to choose $\theta$ to be the set of all subsets of singleton hypotheses. The disadvantage of this approach is its computational problems. An alternative is to apply D-S theory after partitioning the set of hypotheses into groups of mutually exclusive hypotheses (a group of competitive hypotheses), and then consider each group as a separate frame of discernment. This approach, however, requires partitioning rules, which may not be derived easily.

A novel approach was developed in this research for handling multiple virtual links. In this approach, we use the same hypothesis space as before, i.e., the hypothesis space $H$ consists of all potential virtual links. The main differences between this approach and the earlier approach are the formulation of the frame of discernment and the interpretation of the evidences. Unlike the frame in the earlier approach, which contains only all the potential virtual links (only singleton hypotheses). In this approach, $\theta$ is selected to include all subsets of the set of potential virtual links. For the example shown in Fig. 1(b), we may choose $\theta$ to contain all subsets of all possible missing links, i.e., a total of 26 elements in $\theta$, as shown below:

$$
\begin{aligned}
\theta= & \left\{\left\{f_{1} f_{3}\right\},\left\{f_{1} f_{6}\right\},\left\{f_{2} f_{3}\right\},\left\{f_{2} f_{6}\right\},\left\{f_{3} f_{6}\right\},\left\{f_{4} f_{5}\right\},\right. \\
& \left\{f_{1} f_{3}, f_{1} f_{6}\right\},\left\{f_{1} f_{3}, f_{1} f_{6}\right\}, \ldots \\
& \left\{f_{1} f_{3}, f_{1} f_{6}, f_{2} f_{3}\right\},\left\{f_{1} f_{3}, f_{1} f_{6}, f_{2} f_{6}\right\}, \ldots \\
& \left\{f_{1} f_{3}, f_{1} f_{6}, f_{2} f_{3}, f_{2} f_{6}\right\},\left\{f_{1} f_{3}, f_{1} f_{6}, f_{2} f_{3}, f_{3} f_{6}\right\}, \\
& \left\{f_{1} f_{3}, f_{1} f_{6}, f_{2} f_{3}, f_{2} f_{6}, f_{3} f_{6}\right\}, \ldots \\
& \left.\left\{f_{1} f_{3}, f_{1} f_{6}, f_{2} f_{3}, f_{2} f_{6}, f_{3} f_{6}, f_{4} f_{5}\right\}\right\} .
\end{aligned}
$$

One disadvantage with this frame formulation is the computational complexity caused by the huge number of elements in the frame. Given $n$ singleton hypotheses, there would be $2^{n}$ subsets or $2^{n}$ hypotheses included in the frame. An even bigger number may result if we consider the subsets of the $2^{n}$ elements in $\theta$. This problem can be overcome by using domain-specific heuristic knowledge to significantly reduce the scope of the problem, based on the assumption that only certain elements in $\theta$ are of semantic interest to us. Those elements can therefore be selected to form a much smaller frame. For this research, the frame pruning is based on the heuristic assumption that an evidence bearing directly on a hypotheses in the frame points to its presence, while non-evidence on a hypothesis in the frame points to its absence. This also applies to negative evidences since a 
hypotheses with negative evidences should be preserved to evaluate their impacts on other hypotheses. With very limited evidences, this assumption ensures that a large number of hypotheses in the frame would be pruned, resulting in a manageable size of frame of discernment. Consider the frame in Eq. (3) for example, where a frame of this size is not manageable and therefore must be reduced. Based on the pruning assumption and the available evidences, we can prune the elements in the above frame to obtain the new frame as shown below. All hypotheses in the new frames have evidences bearing directly on them.

$$
\begin{aligned}
\theta= & \left\{\left\{f_{1} f_{3}\right\},\left\{f_{1} f_{6}\right\},\left\{f_{2} f_{3}\right\},\left\{f_{2} f_{6}\right\},\left\{f_{3} f_{6}\right\},\left\{f_{4} f_{5}\right\},\right. \\
& \left.\left\{f_{1} f_{3}, f_{2} f_{6}\right\},\left\{f_{1} f_{3}, f_{3} f_{6}\right\},\left\{f_{1} f_{3}, f_{2} f_{6}, f_{3} f_{6}\right\}\right\} .
\end{aligned}
$$

\subsubsection{Feature evidences}

While the combination of the face-based evidences exerting impact upon singleton subset of $\theta$ may sometimes indicate which hypotheses should be preferred over others, the face-based evidences by themselves may be insufficient for deciding the correct choices, because they only carry local information about the object. They are only concerned with the geometry and topology between two faces. Furthermore, the face-based evidences do not allow us to directly exploit the hypotheses at different abstraction levels, because they only interact with hypotheses of the singleton subsets of $\theta$.

Another type of evidence, referred to as feature-based evidences, was therefore developed. Feature-based evidences provide and integrate information which is more global and may encompass one or more potential virtual links. For example, a group of faces may satisfy certain properties which are commonly found in pockets (e.g. in a group of side-faces, forming a loop, each side-face is concavely adjacent to two other neighboring side-faces), then this information about this collection of faces is used by a feature-based evidence to support the hypothesis for those virtual links involved in such a pocket .

Feature-based evidences may simultaneously support one or more virtual links. Consequently, these evidences may exert their impact upon non-singleton subsets of . For instance, in terms of our example object shown in Fig. 1(b), the collection of faces $1-5$ satisfy most properties of pockets including the above stated property that every side-face $(2-5)$ is concavely adjacent to two other side-faces. As a result, the subgraph representing a pocket by this collection of faces constitutes a pocket feature evidence supporting the hypothesis for a virtual link between 1 and 3. It is clear that in supporting or disconfirming a virtual link between a pair of faces, feature-based evidences consider and evaluate the relationships of those faces with their surrounding faces and edges (topologic entities). Thus, they represent and apply information at a higher level of abstraction.

As required by the earlier approach, where each evidence is associated with a bpa to indicate the belief in a hypothe- sis (or a set of hypotheses) given an evidence, a bpa is also required for this approach to indicate the belief in a hypothesis given an evidence. Since feature evidences are positive evidences, bpa's are always assigned directly to links supported by the evidences rather than to their complements. The numerical values of bpa's are determined empirically. Two values of 0.9 and 0.6 are used. While 0.9 is assigned to feature evidences containing only one potential virtual link, 0.6 is assigned to evidences containing more than one potential virtual links. The rational is that there is less ambiguity associated with evidences that have fewer virtual links and therefore higher belief should be assigned to the hypotheses concerned.

\subsubsection{Evidence interpretation through the principle of association}

The frame of discernment shown in Eq. (4) contains both singleton and non-singleton elements. Further, the multi-link (non-singleton) elements in the frame represent the conjunction rather disjunction of its constituents. The available evidences must, therefore, be interpreted differently. A theory of principle of association was developed through this research to assign beliefs to hypotheses in a coherent and consistent manner. The principle of association relates an evidence to all hypotheses that are implied by the evidence. For example, any evidence that confirms the link 1-3 signifies its support for any hypotheses, be it singleton or composite, that contain the link $1-3$ or a proposition set of $\left\{\left\{f_{1} f_{3}\right\}\right.$ or $\left\{f_{1} f_{3}, f_{2} f_{6}\right\}$ or $\left\{f_{1} f_{3}, f_{3} f_{6}\right\}$ or $\left.\left\{f_{1} f_{3}, f_{2} f_{6}, f_{3} f_{6}\right\}\right\}$ for our example part. For this research, this principle of association can be summarized as follows:

- An evidence confirming a link or a set of links in a frame actually commits its belief to a proposition that contains all disjoint elements of the frame that contain the link or the set of links.

- An evidence disconfirming a link or a set of links in a frame actually commits its belief to a proposition that contains all disjoint elements of the frame that do not have the link or the set of links.

Based on these principles, the evidences in our example can redirect their beliefs to proper hypotheses.

\subsubsection{Experimental results}

In this section, we will show an example of the implementation of this new approach to determine multiple virtual links simultaneously. The example part is shown in Fig. 1(b). The hypothesis space $H$ consists of links from the complement of the cavity graph. For the example, $H$ includes links $f_{1} f_{3}, f_{1} f_{6}, f_{2} f_{3}, f_{2} f_{6}, f_{3} f_{6}$, and $f_{4} f_{5}$. The frame of discernment is obtained by including all the subsets of the potential virtual links, followed by a pruning of the initial frame to eliminate the impossible hypotheses as shown in Eq. (4). Both face and feature evidences are applicable to this approach. Therefore, by applying both face 
Table 3

Combined BPAs for the example part shown in Fig. 1(b)

$m_{T}\left(\left\{f_{1} f_{3}, f_{2} f_{6}\right\}\right)=\mathbf{0 . 5 3 2}$

$m_{T}\left(\left\{f_{1} f_{3}\right\},\left\{f_{1} f_{3}, f_{2} f_{6}\right\}\right)=0.039$

$m_{T}\left(\left\{f_{1} f_{6}\right\},\left\{f_{1} f_{3}, f_{2} f_{6}\right\}\right)=0.039$

$m_{T}\left(\left\{f_{1} f_{3}\right\},\left\{f_{1} f_{3}, f_{2} f_{6}\right\},\left\{f_{1} f_{3}, f_{2} f_{6}, f_{3} f_{6}\right\}\right)=0.009$

$m_{T}\left(\left\{f_{2} f_{6}\right\},\left\{f_{1} f_{3}, f_{2} f_{6}\right\},\left\{f_{1} f_{3}, f_{2} f_{6}, f_{3} f_{6}\right\}\right)=0.009$

$m_{T}\left(\left\{f_{1} f_{3}, f_{2} f_{6}\right\},\left\{f_{1} f_{3}, f_{2} f_{6}, f_{3} f_{6}\right\}\right)=0.141$

$m_{T}\left(\left\{f_{1} f_{3}, f_{2} f_{6}, f_{3} f_{6}\right\}\right)=0.231$

evidences and feature evidences to the frame in Eq. (4), by using the principle of association, and then by combining them using Eq. (1), we obtain the combined bpa for the hypotheses shown in Table 3.
Since hypothesis $\left\{f_{1} f_{3}, f_{2} f_{6}\right\}$ has the highest belief committed to it, it is selected and as a result, the most probable virtual links are link $1-3$ and link $2-6$. This result is the same as the results obtained through the earlier approach. By comparison, it is clear that this approach has the advantage of identifying all virtual links simultaneously, greatly reducing the computational complexity.

\section{Another example}

Before we conclude, we would like to show the application our approach to an non-trivial example object and discuss the implementation methods and experimental results. Fig. 6 presents the geometry of the part, along with its cavity

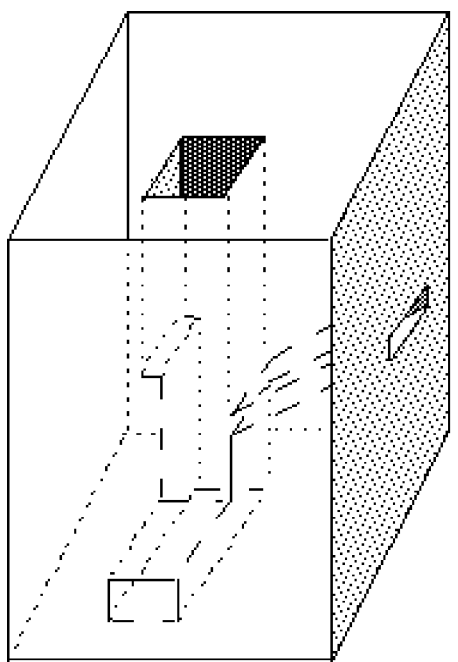

(a1)

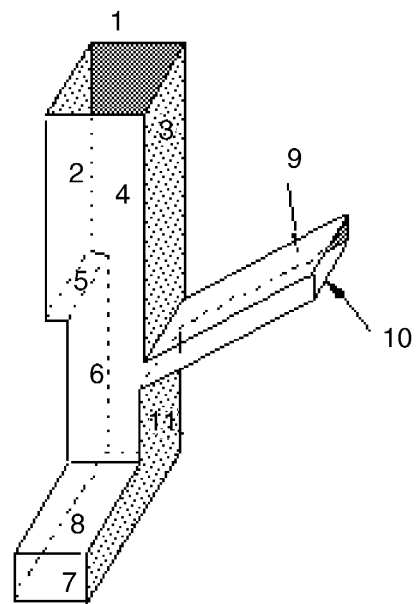

(a2)

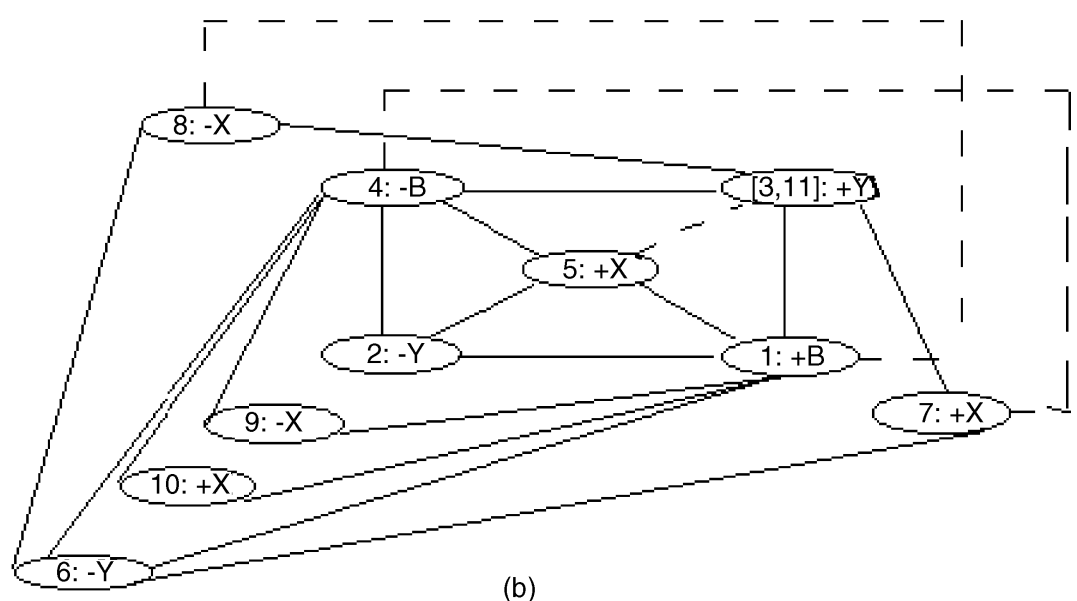

Fig. 6. (a1) and (a2) show an example object with non-trivial feature interactions and its depression. In (b) the solid lines show the cavity graph of the depression, which [3,11] means that two faces 3 and 11 are unified and are represented by one node. Only if the original cavity graph is augmented with three virtual links as shown in (b) by dashed links the form features of the object can be correctly identified and extracted. 
Table 4

All possible single-link hypotheses for the example part shown in Fig. 6(a). $(i, j)$ represents the link formed by face $i$ and face $j$

\begin{tabular}{ll}
\hline Hypotheses & Links \\
\hline$h_{1}$ & $(1,8)$ \\
$h_{2}$ & $(3,5)$ \\
$h_{3}$ & $(4,7)$ \\
$h_{4}$ & $(6,9)$ \\
$h_{5}$ & $(6,10)$ \\
$h_{6}$ & $(1,4)$ \\
$h_{7}$ & $(2,3)$ \\
$h_{8}$ & $(2,6)$ \\
$h_{9}$ & $(2,7)$ \\
$h_{10}$ & $(2,8)$ \\
$h_{11}$ & $(2,9)$ \\
$h_{12}$ & $(2,10)$ \\
$h_{13}$ & $(3,11)$ \\
$h_{14}$ & $(3,9)$ \\
$h_{15}$ & $(3,10)$ \\
$h_{16}$ & $(4,8)$ \\
$h_{17}$ & $(5,6)$ \\
$h_{18}$ & $(5,7)$ \\
$h_{19}$ & $(5,8)$ \\
$h_{20}$ & $(5,9)$ \\
$h_{21}$ & $(5,10)$ \\
$h_{22}$ & $(7,8)$ \\
$h_{23}$ & $(7,9)$ \\
$h_{24}$ & $(7,10)$ \\
$h_{25}$ & $(8,9)$ \\
$h_{26}$ & $(8,10)$ \\
$h_{27}$ & $(9,10)$ \\
\hline &
\end{tabular}

graph representation. A total of 27 single-link hypotheses may be generated. They are given in Table 4 .

The frame of discernment constructed from the above singleton hypotheses is too large $\left(2^{27}\right)$ for effective evidence aggregation. It contains many hypotheses which are useless or irrelevant. Some of the multi-link hypotheses may even represent impossible hypotheses. For example, a hypothesis (e.g., $h_{8}$ ) may include a link resulted from the intersection between two faces (faces 2 and 6) which are geometrically parallel. As a result, the original frame of discernment must be pruned so that only hypotheses with direct evidence are preserved in the new frame of discernment. Both the face-based and feature-based evidences are used for pruning. A total of 37 face evidences and 6 feature evidences were produced. The face-based evidences exert their impact on the singleton link hypotheses (so all singleton hypotheses are preserved) while the feature-based evidences impact both the singleton and multi-link hypotheses.

In order to find and instantiate the applicable feature-based evidences, we followed the following implementation strategy. Different subsets of potential virtual links are added to the original cavity graph of the object, and subsequently the resulting graph is searched for possible features which
Table 5

Combined BPAs for the example part shown in Fig. 6(a)

$$
\begin{aligned}
& m_{T}\left(\left\{h_{1}\right\}\right)=0.63 \\
& m_{T}\left(\left\{h_{2}\right\}\right)=0.63 \\
& m_{T}\left(\left\{h_{3}\right\}\right)=\mathbf{0 . 7 2} \\
& m_{T}\left(\left\{h_{4}\right\}\right)=0.47 \\
& m_{T}\left(\left\{h_{5}\right\}\right)=0.47 \\
& m_{T}\left(\left\{h_{i}\right\}\right)=0.35 \text { for } i=9,10,11,12 \\
& m_{T}\left(\left\{h_{i}\right\}\right)=0.00 \text { for } i=6,7,8,13,18,19, \ldots, 27 \\
& m_{T}\left(\left\{h_{i}\right\}\right)=0.24 \text { for } i=14,15,16,17 \\
& m_{T}\left\{h_{1}, h_{2}\right\}=\mathbf{0 . 8 5} \\
& m_{T}\left\{h_{3}, h_{4}\right\}=0.52 \\
& m_{T}\left\{h_{3}, h_{4}, h_{5}\right\}=0.34
\end{aligned}
$$

contain some of these potential virtual links. If templates for such features are found which contain some of these virtual links, then the involved group of entities (faces and edges) are checked for particular relationships which are often found in a valid feature of the given template type. If these expected relationships exist, then an evidence is instantiated, and the appropriate likelihood assignment is made to support the hypothesis for the involved virtual links. The searching for the templates is an isomorphism algorithm and is implemented as a depth-first tree-search.

Two points should be made about this implementation method for generation of feature-based evidences. First, the evidences should be applied for the maximally possible feature template. For example, if a given virtual link can be supported by both a possible pocket, and a possible blind-slot, then as long as the pocket template subsumes the blind-slot template, we only instantiate a pocket evidence. This method prohibits multiple counting of dependent evidences that could be generated (Since a blind-slot template is a subgraph of a pocket template, it is possible that whenever a pocket template is found, a blind-slot template may also be found, and this could contribute to multiple counting of evidence). Secondly, in order for this depth-first tree-search method to work, we may not add the entire set of potential virtual links to the original cavity graph at once. Rather, at each time we need to add a subset of these potential links to the cavity graph, and search the new structure for possible features. Otherwise, if all nodes in this structure are connected by (potential) links, the template searching operation implemented as a subgraph isomorphism may not find some of the templates, and could produce incorrect results.

The new frame of discernment after pruning is

$$
\begin{gathered}
\theta=\left\{h_{1}, h_{2}, h_{3}, h_{4}, h_{5}, h_{6}, \ldots, h_{27},\left\{h_{1}, h_{2}\right\},\right. \\
\left.\left\{h_{3}, h_{4}\right\}\left\{h_{3}, h_{4}, h_{5}\right\}\right\}
\end{gathered}
$$

After collecting the relevant face-based and feature-based evidences and the combination of the collected evidences over frame $\theta$, the combined BPAs for each hypothesis in $\theta$ are given in Table 5 . 
Since hypotheses $\left\{h_{1}, h_{2}\right\}$ and $h_{3}$ have the highest belief committed to them, they are selected and as a result, the most probable virtual links are links $1-8,3-5$, and 4-7. Augmenting the original cavity graph with these links and partitioning it correctly identifies the form features for the object in Fig. 6.

\section{Conclusion}

The main contributions of this research work can be summarized as introducing a method to represent and model knowledge with uncertainty in feature recognition, developing different classes of evidences based on the geometric and topologic relationships at different abstraction levels for effective reasoning, and developing the principle of association, which enables us to identify multiple virtual links simultaneously by overcoming the mutual exclusiveness assumption of the Dempster-Shafer theory. Development of methods such as those described here is important for overcoming the non-uniqueness in topologic and geometric description of various features in different interactions. The non-uniqueness of feature descriptions in different interactions has been one of the major hindrances in automated identification and extraction of features from CAD solid models. Although the proposed methods can successfully extract the interacting features for many parts, much work still remains to be done. One immediate goal for us is to generalize the developed methodology to identify and extract features with curved surfaces from components with non-planar surfaces.

\section{References}

[1] M.R. Henderson, Computer recognition and extraction of form features: a cad/cam link, Comput. Ind. 5 (1984) 329-339.

[2] M.R. Joshi, T.C. Chang, Graph-based heuristics for recog nition of machined features from a 3-d solid model, Comput. Aided Des. 20 (2) (1988) 58-66.

[3] H. Kung, Investigation into development of process plans from solid geometric modeling representation, Ph.D. Thesis, Oklahoma State University, 1984.

[4] L.K. Kyprianou, Classification in computer-aided design, $\mathrm{Ph} . \mathrm{D}$. Thesis, University of Cambridge, Cambridge, England, 1980.

[5] M.M. Marefat, R.L. Kashyap, Geometric reasoning for recognition of three dimensional object features, IEEE Trans. Pattern Anal. Mach. Intell. 12 (10) (1990), 949-965.

[6] S.M. Staley, Using syntactic pattern recognition to extract feature information from a solid geometric data base, Comput. Mech. Eng. 2 (2) (1983) 61-66.

[7] T.C. Woo, Feature extraction by volume decomposition, Proceedings of Conference in CAD/CAM Technology in Mechanical Engineering, MIT, Cambridge, MA, 1982.

[8] Shu-Chu Liu, M. Gonzalez, Development of an automatic part feature extraction and classification system taking cad data as input, Comput. Ind. 29 (3) (1996) 137-150.
[9] J. Dong, S. Vijayan, Near optimal approach to general manufacturing feature extraction, ASME Des. Eng. (DE) 83 (2) (1995) 809-816.

[10] C.Y. Kao, Soundar R.T. Kumara, Extraction of 3d object features from cad boundary representation using the super relation graph method, IEEE Trans. Pattern Anal. Mach. Intell. 17 (12) (1995) 1228-1233.

[11] A.Z. Qamhiyah, R.D. Venter, Geometric reasoning for the extraction of form features, Comput. Aided Des. 28 (11) (1996) 887-903.

[12] R. Ganesan, V. Devarajan, Approach to extracting intersecting features from 2d cad, ASME Des. Eng. (DE) Publ. 94 (1997) 79-94.

[13] R. Ganesan, V. Devarajan, Intersecting features extraction from $2 \mathrm{~d}$ orthographic projections, Comput. Aided Des. 30 (11) (1998) 863-873.

[14] J.Y. Lee, K. Kim, Feature-based approach to extracting machine features, CAD Comput. Aided Des. 30 (13) (1998) 1019-1035.

[15] K.S. Fu, Syntactic Pattern Recognition and Applications, Prentice-Hall, Englewood, Cliffs, NJ, 1982.

[16] R. Li, A conceptual framework for the interaction of computer-aided design and computer-aided manufacturing, Ph.D. Thesis, Arizona State University, 1986.

[17] G. Falcidieno, F. Fiannini, Automatic recognition and representation of shaped-based features in a geometric modeling system, Comput. Vision, Graph. Image Process. 48 (10) (1989) 93-123.

[18] E. Wang, Using automatic feature recognition to interface cad to capp, Proceedings of ASME Computers in Engineering, San Francisco, CA, 1992.

[19] L. DeFloriani, Feature extraction from boundary models of 3d objects, IEEE Trans. PAMI 11 (10) (1989) 785-798.

[20] M.R. Henderson, S.H. Chuang, G.P. Gavankar, Graph-based feature extraction, in: Proceedings of NSF Design and Manufacturing Systems Conference, Tempe, AZ, 1990, pp. 183-189.

[21] J. Corney, D. Clark, Method for finding holes and pockets that connect multiple faces in $21 / 2 \mathrm{~d}$ objects, Comput. Aided Des. 20 (10) (1990) 658-668.

[22] M.C. Fields, D.C. Anderson, Fast feature extraction for machining applications, Comput. Aided Des. 26 (11) (1994) 803-813.

[23] A.A.G. Requicha, Geometric reasoning for intelligent manufacturing, Commun. ACM 39 (2) (1996) 71-76.

[24] K.E. Hummel, Coupling rule-based and object oriented programming for the classification of machine features, in: Proceedings of ASME Conference on Computers in Engineering, Vol. 1, 1989, pp. 409-418.

[25] S.N. Trika, R.L. Kashyap, Geometric reasoning for extraction of manufacturing features in iso-oriented polyhedron, in: Proceedings of ASME Conference on Computers in Engineering, 1994, pp. 1087-1100.

[26] M. Marefat, Q. Ji, Hierarchical Bayesian methods for recognition and extraction of $3 \mathrm{~d}$ features from cad solid models, IEEE Trans. Systems, Man, Cybernet. 7 (6) (1997).

[27] J. Pearl, Probabilistic Reasoning in Intelligent Systems, Morgan Kaufmann, Palo Alto, CA, 1989.

[28] G. Shafer, A Mathematical Theory of Evidence, Princeton University Press, Princeton, NJ, 1976. 
[29] David Waltz, Understanding line drawing of scences with shadows, in: P.H. Winston (Ed.), The Psychology of Computer Vision, Cambridge University Press, Cambridge, 1975, pp. 19-91.
[30] A.K. Mackworth, Consistency in networks of relations, Artif. Intell. 8 (1) (1977) 99-118.

[31] G.A. Shafer, A Mathematical Theory of Evidence, Princeton University Press, Princeton, NJ, 1979.

About the Author-QIANG JI received a MS degree in electrical engineering from the University of Arizona in 1993 and his Ph.D. degree in Electrical Engineering from the University of Washington in 1998. He is currently an Assistant Professor with the Department of Electrical, Computer, and Systems engineering at Rensselaer Polytechnic Institute. Previously, he was an assistant Professor with the Department of Computer Science at University of Nevada at Reno.

Dr Ji's research areas include computer vision, image processing, pattern recognition, information fusion, and robotics. Dr. Ji has published more than 50 papers in referred journals and conferences in these areas. His research has been funded by local and federal government agencies such as NIH, AFOSR, and ARO and by private companies including Boeing and Honda.

About the Author-MICHAEL M. MAREFAT received the B.S. degree in Electrical and Computer Engineering and the B.A. degree in mathematical sciences from Rice University, Houston, TX, in 1986. He received the M.S. and Ph.D. degrees in Electrical Engineering from Purdue University, West Lafayette, IN, in 1988 and 1991, respectively.

He is the Director of the Knowledge Systems Engineering Laboratory and a Faculty Member in the Department of Electrical and Computer Engineering at the University of Arizona, Tucson. He has been involved in research in the areas of knowledge-based systems, visualization, computer graphics, machine vision, and CAD for the last 15 years. He is the author or co-author of over 100 refereed research articles. His research has been supported by the National Science Foundation and by NASA. His previous experience includes Schlumberger Laboratory for Computer Science, Purdue Engineering Research Center for Intelligent Manufacturing Systems, and Baylor Digital Imaging Laboratory. 